\title{
Patient and caregiver perceptions of a pre-filled diluent syringe (MixPro®)
}

Jim Munn*, Kate Khair, Andrew Scott, Robyn Shoemark, Julia Spires, Morten Lind Jensen, Reto Wirz

Prophylactic coagulation factor replacement is increasingly the treatment modality of choice for people with haemophilia (PWH). Currently available recombinant factor products require reconstitution from a lyophilised powder and diluent, and a range of infusion systems is available to assist in this process. This study aimed to understand the properties of a reconstitution/infusion system that are most important to PWH and carers of children with haemophilia (CWH), and to assess two available systems produced by Novo Nordisk for the reconstitution and infusion of activated recombinant factor VII and recombinant factor VIII: the original infusion system and the newer MixPro ${ }^{\circledR}$ system. Both were tested by a group of $67 \mathrm{PWH}$ or carers of CWH who were naïve to them. Participants rated the performance of each system against 18 predefined parameters using the 7-point Likert scale, and ranked the importance of these parameters to the design of an infusion system. They also directly compared the performance of the two systems and provided qualitative feedback. Overall, MixPro ${ }^{\circledR}$ was preferred to the original system by $94 \%$ of study participants. This was reflected in the performance scores for individual parameters, with scores in 16/18 parameters being significantly higher for MixPro $®(p<0.05)$ than the original system. Low contamination risk was seen as the most important criterion in the design and choice of an infusion system, with $97 \%$ regarding MixPro® as the superior system in this category. The MixPro ${ }^{\circledR}$ system was perceived as being quick, easy to use, convenient and portable. It is hoped that these findings may help guide the future design of infusion systems for PWH.

Keywords: recombinant Factor VIla, haemophilia, prophylactic treatment, infusion system

Haemophilia is a serious genetic condition that impairs the blood's ability to clot, due to a deficiency in one or more

* James Munn, University of Michigan Hemophilia and Coagulation Disorders Program, University of Michigan, 1500 E. Medical Center Drive, Ann Arbor, MI, USA. Email: jmunnamed.umich.edu

Kate Khair, Haemophilia Centre, Great Ormond Street Hospital for Children NHS Trust, London, UK

Andrew Scott, Phoenix Healthcare, London, UK

Robyn Shoemark, The Children's Hospital at Westmead, Sydney, Australia Julia Spires, Haemophilia Centre, Great Ormond Street Hospital for Children NHS Trust, London, UK

Morten Lind Jensen, Novo Nordisk A/S, Copenhagen, Denmark Reto Wirz, Novo Nordisk Health Care AG, Zürich, Switzerland

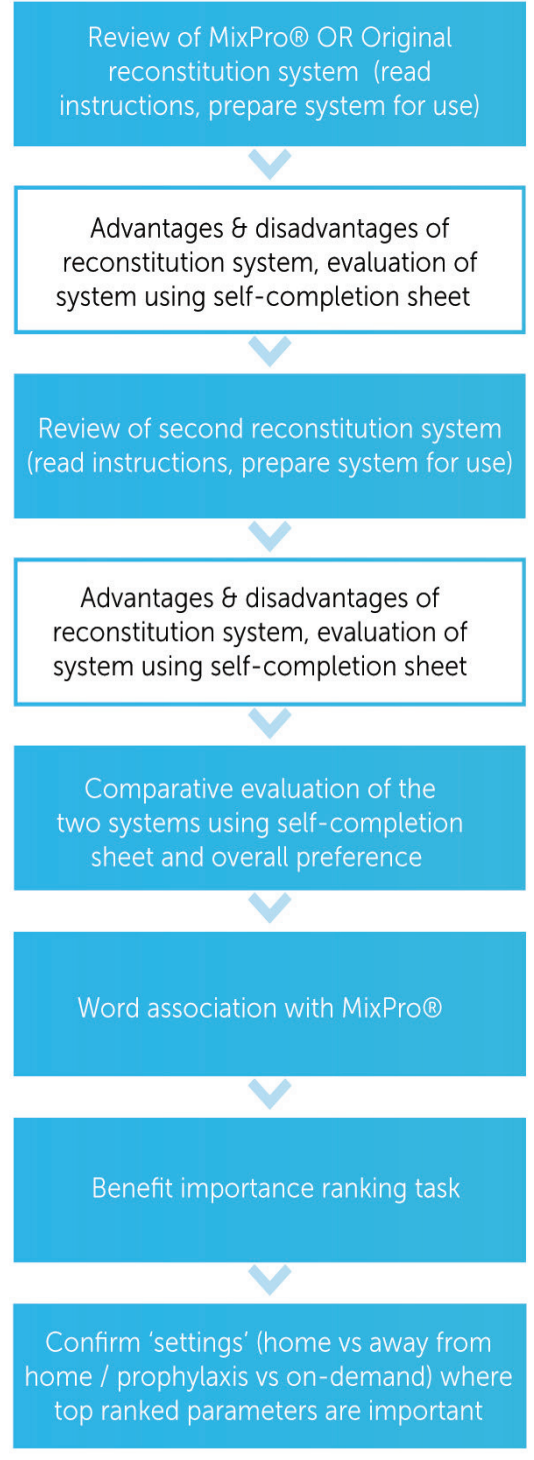

Figure 1. Sequence of steps completed by study participants

blood coagulation plasma proteins, also called coagulation factors. People with haemophilia (PWH) are potentially prone to severe bleeding from even minor injuries [1]. Prophylactic factor replacement is the treatment modality of choice for those with severe haemophilia without inhibitors or mild-moderate haemophilia with a phenotype 
predisposing to bleeding, particularly in Western Europe and the USA $[2,3]$. Both prophylaxis and on-demand/ episodic treatment require intravenous administration of recombinant or plasma-derived replacement factor over the long term, usually carried out by PWH themselves.

Currently available recombinant replacement concentrates require preparation of the infusion solution from a lyophilised powder using a diluent and an infusion system specific to the product. Infusion systems vary in their design and method of use, but generally consist of one or more vials containing factor and diluent, and at least one syringe for the final administration. The reconstitution and infusion process can be time- and labour-intensive. Some system-related concerns previously cited by PWH include inconvenience, needle-stick injuries, loss of factor, length of time to reconstitute factor or infuse, and difficulty in using the system [4-9]. The design and ease of use of infusion systems can therefore play a key role in improving prophylactic adherence [4, 7], as well as helping PWH to react promptly and confidently to bleeds.

The majority of PWH learn to self-infuse at a young age, becoming self-sufficient by the age of $12-13$ years $[10,11]$. Until that time, carers of children with haemophilia $(\mathrm{CWH})$ are often responsible for infusing replacement factor. Understanding the preferences and challenges faced by $\mathrm{PWH}$ and carers of $\mathrm{CWH}$ when using these systems may improve the design of new delivery systems and hence the efficacy of treatment.

The aim of this study was to explore the perspectives of adult PWH and of carers of $\mathrm{CWH}(<18$ years of age) with respect to the use of infusion systems, looking at the parameters regarded as most important to their design. The study focused on two systems used for activated recombinant factor VII (rFVIla, eptacog alfa activated) and recombinant factor VIII (rFVIII, turoctocog alfa), produced by Novo Nordisk: the original infusion system and the new MixPro ${ }^{\circledR}$ system. The key difference between these systems is how the diluent is stored and mixed with the factor. The original system included lyophilised factor and diluent in two separate vials and required the diluent to be transferred from one vial to the other in order to prepare the factor. The infusion solution was then re-drawn into the syringe before infusion. By contrast, MixPro ${ }^{\circledR}$ includes a syringe pre-filled with diluent, reducing the number of handling steps.

\section{Methods}

\section{Participants}

Adult $\mathrm{PWH}$ ( $\geq 18$ years) and adult carers of a $\mathrm{CWH}<18$ years of age were included in the study. Individuals were deemed to be eligible if they regularly infuse replacement factor at home. Those with previous experience with rFVIla (Novo Nordisk) or either of the two systems used in the study were excluded. Participants were recruited in Italy, Spain and the USA.

\section{Research design}

A pilot survey consisting of interviews with four $\mathrm{PWH}$ and caregivers of $\mathrm{CWH}$ was conducted in the UK, in order to identify criteria by which infusion systems could be rated. The interviews identified 18 parameters that were deemed important in the design and usability of an infusion system:

- Easy to learn how to use the system

- When drawing mixed factor into the syringe, there is good visibility of the factor

\section{Table 1. Summary of study participant demographics}

\begin{tabular}{|c|c|c|c|c|}
\hline Parameter & $\begin{array}{l}\text { Italy } \\
(n=20)\end{array}$ & $\begin{array}{l}\text { Spain } \\
(n=20) \dagger\end{array}$ & $\begin{array}{l}\text { USA } \\
(n=27)\end{array}$ & $\begin{array}{l}\text { Total } \\
(\mathrm{N}=67)\end{array}$ \\
\hline Mean age, years & 29 & 22 & 30 & 27 \\
\hline PWH & 45 & 31 & 38 & 38 \\
\hline $\mathrm{CWH}$ & 13 & 13 & 14 & 13 \\
\hline \multicolumn{5}{|l|}{ Type of haemophilia, A/B } \\
\hline A & $90 \%$ & $80 \%$ & $81 \%$ & $84 \%$ \\
\hline B & $10 \%$ & $20 \%$ & $19 \%$ & $16 \%$ \\
\hline \multicolumn{5}{|l|}{ Treatment type } \\
\hline On demand & $45 \%$ & $25 \%$ & $15 \%$ & $27 \%$ \\
\hline Prophylaxis & $55 \%$ & $75 \%$ & $85 \%$ & $73 \%$ \\
\hline \multicolumn{5}{|l|}{ Current treatment* } \\
\hline Factor VIII recombinant & $85 \%$ & $55 \%$ & $78 \%$ & $73 \%(75 \%)^{*}$ \\
\hline Factor VIII plasma derived & $5 \%$ & $20 \%$ & $4 \%$ & $9 \%(25 \%)^{*}$ \\
\hline Factor IX recombinant & $5 \%$ & $10 \%$ & $19 \%$ & $12 \%(13 \%)^{*}$ \\
\hline Factor IX plasma derived & $5 \%$ & $10 \%$ & $0 \%$ & $4 \%(7 \%)^{*}$ \\
\hline
\end{tabular}

*Current use/ever used provided in brackets (note: percentages may not total 100\% due to rounding error). Question: "Which replacement factor product are you/is your son currently using to treat your/his hemophilia?" (Base: all people with hemophilia/caregivers). ${ }^{\dagger}$ One patient was being treated with activated prothrombin complex concentrate (APCC) at the time of the study. 
- Very portable

- Low contamination risk when mixing

- Easy handling steps

- Low number of handling steps during mixing

- Low number of separate parts

- Easy to use if you need more than one vial for an infusion

- Suitable for a person with less strength (e.g. child, elderly, etc)

- I am confident I could use the system correctly

- System is intuitive to use

- System is convenient to use

- Easy to teach someone else how to use the system

- Mixing can be accomplished quickly

- Easy to verify that all the mixed factor has been drawn into the syringe

The system is sturdy

- I feel in control of the mixing process

- Overall the system is easy to use

The sequence of assessments completed by respondents in this study is detailed in Figure 1. Briefly, study participants were asked to use each system in turn to simulate the preparation of an inactive test medium for infusion, using only the instructions provided. The order in which the systems were tested was reversed at alternate interviews in order to eliminate bias. After each test, participants rated the system against the 18 parameters on a Likert scale of 1 (does not describe at all) to 7 (completely describes), using a self-completion sheet.

A structured 30-minute face-to-face interview was then conducted with each participant, in which they described the reasons for their decisions and their overall preference according to the question: "Overall, taking everything into account, which of the two systems do you prefer the most?" Participants were then asked to state which of a predefined list of words they most associated with the MixPro $₫$ system, after having used it for the first time. Finally, they were asked to rank the 18 parameters in order of importance to the design of an infusion system, and state whether they considered them to be 'more important', 'less important' or 'equally important' in different settings: infusing at home vs infusing away from home, and preparing an infusion for prophylaxis vs preparing an infusion to treat a bleed.

\section{Data analyses}

Ratings across the 18 parameters for each system were compared in terms of the mean score on the 7-point Likert scale and the percentage of respondents choosing a top score (6 or 7) for each parameter. The Z-test was used to identify significant differences at a $95 \%$ level of confidence.

An overall importance score for each of the 18 parameters was computed by compiling a 'win-loss' table, showing the frequency with which one parameter was ranked as more important than each other parameter. These data were entered into a scaling algorithm that calculated an importance score for each parameter. The importance

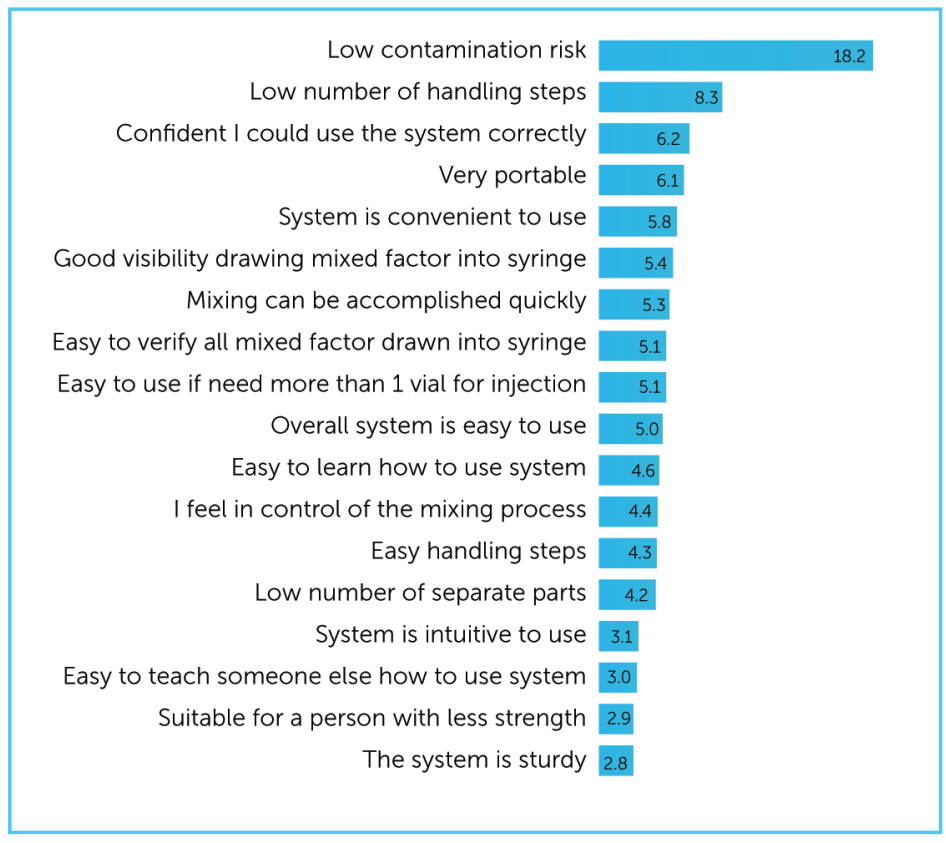

Figure 2. Respondent importance scores and ranking of 18 predefined parameters of an infusion system. Participants ranked the 18 parameters in order of their perceived importance to the design and choice of an infusion system. An overall importance score for each parameter was computed by compiling a 'win-loss' table, showing the frequency with which one parameter was ranked as more important than each other parameter. These data were entered into a scaling algorithm that calculated an importance score for each parameter. The importance score is a linear measure: for example, a score of 20 is perceived to be twice as important as one with a score of 10. Respondents rated low contamination risk as the most important of the 18 identified parameters that influence the design and choice of infusion system

score is linear; a parameter with a score of 20 would be twice as important/desired as one with a score of 10 .

\section{Results}

The study was conducted between 17 September 2014 and 24 October 2014. A total of 67 participants (38 PWH and 29 carers of $\mathrm{CWH}$ ) completed the study tasks: 20 from Italy, 20 from Spain and 27 from the USA (Table 1). The PWH participating in this study had a mean age of 38 years, while $\mathrm{CWH}$ being cared for by adult participants had a mean age of 13 years. The majority (84\%) of cases were haemophilia $A$ and were receiving treatment as prophylaxis (73\%). Most $\mathrm{PWH}$ and $\mathrm{CWH}$ with haemophilia A were treated with $\mathrm{rFVIII}$ $(88 \%)$, while most of those with haemophilia B were treated with $\mathrm{rFIX}(73 \%)$. However, a wide range of recombinant and plasma-derived factor replacement products was represented across the study population.

\section{Assessment parameters}

Participants rated 'low contamination risk when mixing' as the most important parameter when considering an infusion system (importance score: 18.2), followed by 'low 


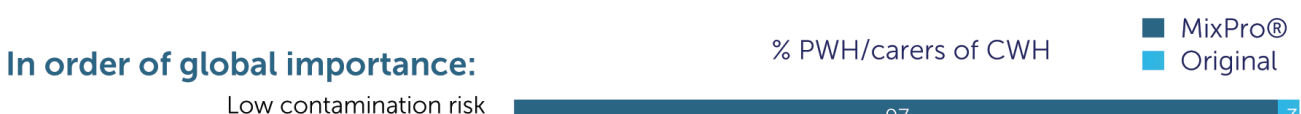

Low number of handling steps

Confident I could use the system correctly

Very portable

System is convenient to use Good visibility drawing mixed factor into syringe Mixing can be accomplished quickly Easy to verify all mixed factor drawn into syringe Easy to use if need more than 1 vial for injection Overall system is easy to use Easy to learn how to use system I feel in control of the mixing process Easy handling steps Low number of separate parts

System is intuitive to use

Easy to teach someone else how to use system Suitable for a person with less strength The system is sturdy

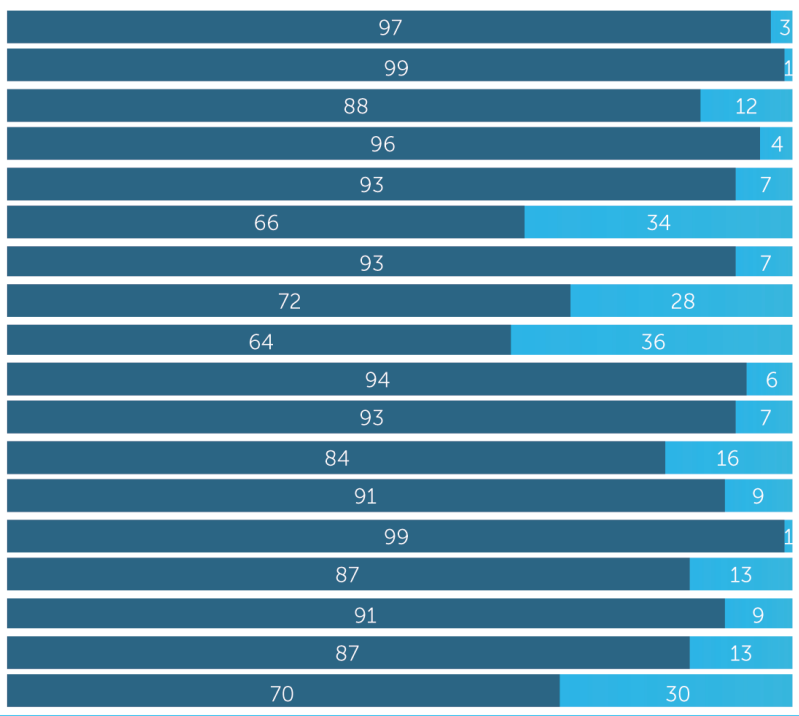

Figure 3. Participant system preference based on the list of 18 predefined parameters. The majority of respondents preferred the MixPro ${ }^{\circledR}$ system to the original infusion system both overall and when assessed on each individual parameter. Question: "Next, I would like you to compare both System O and System M on the characteristics that we have been talking about. For each characteristic please indicate by ticking or checking in the appropriate box which of the two mixing systems you think performs best"

number of handling steps during mixing' (importance score: value on the usability of a system for a person with less 8.3) (Figure 2). Ratings given by PWH vs carers, and by those strength (ranked 3rd by carers vs 18th by PWH).

treated on demand vs prophylactically, were generally comparable (data not shown). PWH placed more emphasis Most parameters were regarded as equally important than carers of $\mathrm{CWH}$ on portability (ranked 3rd by PWH vs whether infusing at home or away from home by the majority 18th by carers) and convenience (ranked 4th by PWH and of respondents (data not shown). The parameters that were 12th by carers). By contrast, carers of $\mathrm{CWH}$ placed greater regarded as being most important when infusing away from

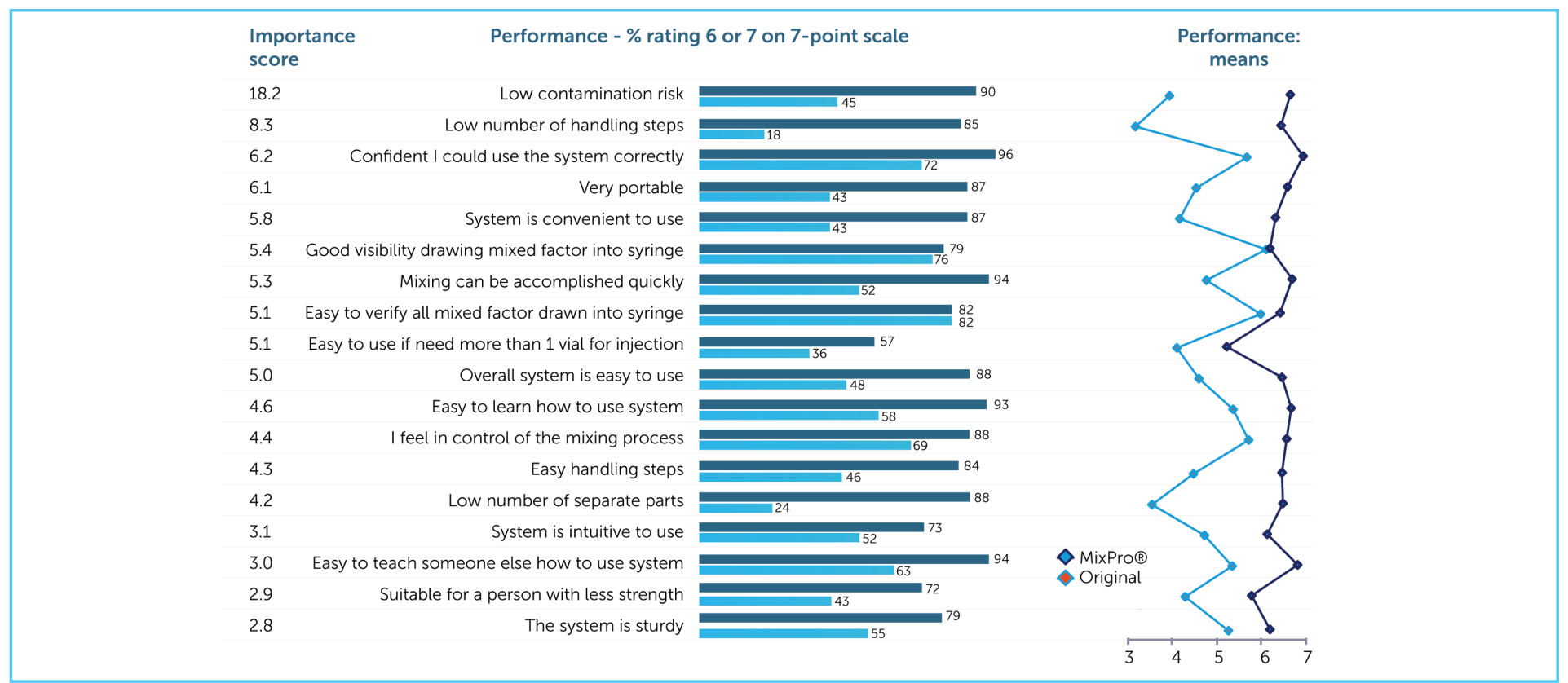

Figure 4. Mean performance score and percentage of respondents selecting a top score (6 or 7 out of 7 ) on the performance scale for each of the 18 predefined parameters assessed across both systems. PWH and carers significantly favoured MixPro ${ }^{\circledR}$ over the original infusion system across 16/18 tested parameters. The only parameters where MixPro ${ }^{\circledR}$ was not significantly favoured were 'good visibility drawing mixed factor into syringe' and 'easy to verify all mixed factor drawn into syringe' 


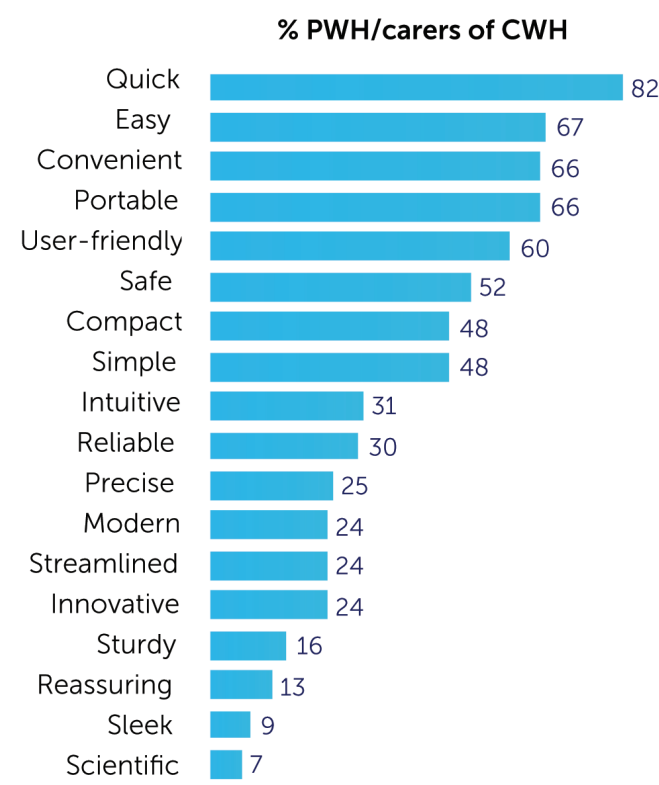

Figure 5. The proportion of respondents who associated a set of words with the MixPro ${ }^{\circledR}$ system based on their experience in the study. The words 'quick', 'easy', 'convenient' and 'portable' were chosen by at least two-thirds of study participants. Question: "Please look through the words on this card and then select a maximum of $\mathbf{8}$ words that you would most associate with System M"

home (compared with at home) included portability (74\%), system sturdiness (57\%), low number of separate parts (35\%) and speed of mixing (33\%).

When considering the relative importance of infusion system properties when used for prophylaxis vs treatment of a bleed, speed of operation (55\%) and ease of teaching third parties to use the system (38\%) were those most cited as being more important for on-demand treatment.

\section{Evaluation of MixPro $₫$ and the original infusion system}

Nearly all participants (94\%) preferred MixPro ${ }^{\circledR}$ over the original system; this was consistent across participants from Italy $(100 \%)$, Spain $(90 \%)$ and the USA (93\%). The strong overall preference for MixPro ${ }^{\circledR}$ was reflected in the per-parameter preferences; MixPro ${ }^{\circledR}$ was preferred by significantly more respondents than the original system when assessing each parameter individually (Figure 3).

The mean performance scores for MixPro ${ }^{\circledR}$ were significantly higher than those for the original system in 16 out of the 18 categories: 'good visibility drawing mixed factor into syringe' and 'easy to verify all mixed factor has been drawn into syringe' were the only two that were not statistical superior (Figure 4). Ratings conferred by PWH and carers of $\mathrm{CWH}$ were almost identical, with no statistically significant inter-group differences in any category (data not shown).
These trends were also reflected in the percentage of respondents assigning top scores (6 or 7 on the 7-point Likert scale), with MixPro ${ }^{\circledR}$ outperforming the original system in the same 16/18 categories (Figure 4). Twice as many participants gave MixPro ${ }^{\circledR}$ a top score for the parameter deemed most important by participants - low contamination risk - compared with the original system (90\% vs $45 \%)$; almost all participants (97\%) also stated that MixPro ${ }^{\circledR}$ performed better than the original system for this parameter (Figure 3). Most participants (96\%) were confident that they could use the MixPro ${ }^{\circledR}$ system correctly, while $73 \%$ thought that it was intuitive to use, and $93 \%$ found it easy to learn how to use (Figure 4).

Importance ranking and performance rating results were generally consistent across sub-populations of PWH vs caregivers and those treated on demand vs prophylactically (data not shown). Due to the sizes of the sub-populations, it was not possible to conduct meaningful country specific sub-analyses.

\section{Qualitative feedback}

At least two-thirds of participants associated MixPro ${ }^{\circledR}$ with being quick, easy, convenient and portable, while at least half found it to be user-friendly and safe (Figure 5). Words with negative connotations, such as 'bulky', 'complicated', 'confusing', 'awkward', 'impractical', 'cumbersome' and 'difficult', were each selected by $<3 \%$ of participants. Qualitative feedback from participants cited fewer constituent parts, faster operation, ease of preserving sterility and avoidance of contamination as positive aspects of the MixPro ${ }^{\circledR}$ system. The potential need for multiple vials/syringes per dose for some people, and difficulty in seeing the liquid were cited as areas for future development. By contrast, qualitative feedback on the original system highlighted the larger transparent syringe capacity compared to MixPro ${ }^{\circledR}$, and allowing the injection of more factor with good visibility as positives. However, the number of steps and length of time needed to operate the original system were criticised, as was the perceived contamination risk.

\section{Discussion}

Overall, MixPro ${ }^{\circledR}$ was perceived favourably by respondents and was regarded as having statistically superior performance compared with the original infusion system in almost all aspects. The only criteria where MixPro ${ }^{\circledR}$ was not clearly rated better than the original system related to verifying that the mixed factor had been drawn into the syringe (Figure 4). Notably, over $90 \%$ of participants preferred MixPro® to the original system in four out of the five parameters that were rated as most important when considering an infusion system. In the top two categories, 97\% preferred MixPro ${ }^{\circledR}$ when considering contamination risk, and $99 \%$ preferred it in terms of low number of handling steps.

The choice of contamination risk as the most important 
property of an infusion may have been influenced by several factors, such as the demographics of the study population. The results would have been expected to show more focus on convenience than contamination if $\mathrm{CWH}$ rather than their carers had been included, since previous surveys using other systems found that $\mathrm{CWH}$ tend to prefer convenient designs $[9,12]$. It is reasonable to assume that MixPro ${ }^{\circledR}$ would be expected to do well on these parameters compared with the original system, as evidenced by the 99\% preference regarding low number of handling steps and the $88 \%$ preference in terms of confidence that people could use the system correctly (Figure 3). Generational differences in what constitutes 'contamination risk' and 'hygiene' may also have been a factor.

The choice of comparator may additionally have influenced the perceived importance of each parameter, drawing attention to those in which the two systems differed the most. Using a wider range of infusion systems may, therefore, have yielded different results.

The multinational design of the study, and the fact that the results were comparable across countries, confers a degree of confidence that the results would be replicated more generally across the haemophilia community. Similarly, the inclusion of a range of recombinant factor products and treatment modalities among the current regimens of the population reflects a wide cross-section of experiences, and can be seen as a study strength. However, although there was adequate power to show differences between the two systems, the small sample size limited the significance of any between-country and between-population differences.

A key limitation was the lack of $\mathrm{CWH}$ in the study population, as they may have had different perspectives to their carers or adult PWH. CWH have been included in previous surveys, suggesting that it is feasible for them to participate. Some bias may also have been introduced due to the participants being volunteers.

This study reaffirms the importance of continued innovation of infusion system design in collaboration with the haemophilia community, and of taking into account the views of system users early in the design process. Improving ease of use and alleviating concerns about safety or contamination may be expected to help $\mathrm{PWH}$ and $\mathrm{CWH}$ adhere to their treatment [4].

A key priority for future research should be the inclusion of $\mathrm{CWH}$ (or parents of newly diagnosed $\mathrm{PWH}$ who have never been exposed to any infusion systems) in the study population, as well as the testing of a wider range of infusion systems, if feasible. It would also be of interest to more rigorously assess whether there are any important geographical variations, based on healthcare practices or local cultures.

\section{Conclusion}

Low contamination risk was seen as the most important criterion in the design and choice of an infusion system, followed by a low number of handling steps. Almost all respondents in this study preferred MixPro ${ }^{\circledR}$ over the original system; this was the case both overall and in each of the 18 individual parameters assessed, including those rated as being the most important. The MixPro ${ }^{\circledR}$ system was perceived as being quick, easy to use, convenient and portable. It is hoped that these findings may help inform the future design of infusion systems for $\mathrm{PWH}$.

\section{Acknowledgements}

Editorial assistance in the preparation of this manuscript was provided by AXON Communications, and was financially supported by Novo Nordisk in compliance with international guidelines for good publication practice. The research described in this article was undertaken by Phoenix Marketing International and funded by Novo Nordisk Health Care AG, Zürich, Switzerland.

This is an Open Access article distributed under the terms of the Creative Commons Attribution License (http://creativecommons.org/licenses/ by/2.0), which permits unrestricted use, distribution, and reproduction in any medium, provided the original work is properly cited.

\section{References}

1. Srivastava A, Brewer AK, Mauser-Bunschoten EP, et al, Guidelines for the management of hemophilia. Haemophilia 2013 19(1): e1-e47.

2. Chambost H, Ljung R, and the Pednet Group. Changing pattern of care of boys with haemophilia in western European centres. Haemophilia 2005 11(2): 92-9.

3. Ljung R, Prophylactic therapy in haemophilia. Blood Rev 2009 23(6): $267-74$.

4. Vidovic N, Musso R, Klamroth R, et al. Postmarketing surveillance study of Kogenate Bayer with Bio-Set in patients with haemophilia A: evaluation of patients' satisfaction after switch to the new reconstitution system. Haemophilia 2010 16(1): 66-71.

5. Musso R, Santoro R, Coppola A, et al. Patient preference for needleless factor VIII reconstitution device: the Italian experience. Int J Gen Med 2010. 3: 203-8.

6. Saxena, K., Barriers and perceived limitations to early treatment of hemophilia. J Blood Med 2013. 4: 49-56.

7. De Moerloose P, Urbancik W, Van Den Berg HM, Richards M. A survey of adherence to haemophilia therapy in six European countries: results and recommendations. Haemophilia 2008 14(5): 931-8.

8. Hacker MR, Geraghty S, Manco-Johnson M, Barriers to compliance with prophylaxis therapy in haemophilia. Haemophilia 2001. 7(4): 392-6. 9. Khair K, Evaluating a self infusion device for children with haemophilia. Paediatr Nurs 2006 18(10): 19-20.

10. Lindvall K, Colstrup L, Wollter IM, et al. Compliance with treatment and understanding of own disease in patients with severe and moderate haemophilia. Haemophilia 2006 12(1): 47-51.

11. Schrijvers LH, Beijlevelt-van der Zande M, Peters $M$, et al. Learning intravenous infusion in haemophilia: experience from the Netherlands. Haemophilia 2012 18(4): 516-20.

12. Khair K. Children's preferences of transfer devices for reconstitution of factors VIII and IX for the treatment of haemophilia. Haemophilia 2009 15(1): 159-67. 